\title{
Sternal Tuberculosis: An Uncommon Presentation
}

Manish Narang', Archana Dwivedi', Shiva Narang ${ }^{2}$, Gopesh Mehrotra ${ }^{3}$

Department of Pediatrics', Medicine ${ }^{2}$ and Radiodiagnosis ${ }^{3}$

University College of Medical Sciences and GTB Hospital,

\section{Abstract:}

Primary tubercular osteomyelitis of sternum presenting as an acute swelling over chest is an extremely rare presentation in pediatric population. We herein report such a case which was diagnosed by CT scan showing lytic lesion and aspiration revealed positive culture for Mycobacterium tuberculosis. Child improved with antitubercular treatment.

Key words: Tubercular osteomyelitis, Sternum

\section{Introduction}

Skeletal tuberculosis as an extrapulmonary entity is uncommon accounting for less than $7 \%$ cases [1]. Tubercular involvement of the sternum is rarer even in countries where tuberculosis is highly prevalent[2]. We herein report an unusual case of tuberculous osteomyelitis of sternum with acute presentation as a swelling over anterior chest wall.

\section{Case Report}

An eleven year old girl presented with one week history of chest pain and swelling over anterior chest wall. There was history of progressive weight loss and low grade intermittent fever for one month. She was BCG vaccinated and there was no history of contact with tuberculosis in the family.

On examination, the child was febrile, with thin built, and weighed $35 \mathrm{kgs}$ (<50th percentile). Local examination showed diffuse swelling over manubrium. The swelling was highly tender and fluctuant. There was no redness, localised rise of temperature or any venous prominence over the swelling. There was no lymphadenopathy. Systemic examination revealed dullness over bilateral lower chest with decreased breath sounds on corresponding areas. Other systems were found to be normal.

Investigations revealed hemoglobin of $10.4 \mathrm{gm} / \mathrm{dl}$, erythrocyte sedimentation rate was elevated to $40 \mathrm{~mm}$ in $1 \mathrm{st}$ hour. Tuberculin test was strongly positive measuring $20 \times 20 \mathrm{~mm}$ with negative HIV test. Chest x-ray showed bilateral pleural effusion. Computed tomography of the chest showed permeative destruction (lytic areas) of sternum along with extrapleural collection tracking all along the anterior mediastinum and encroaching on anterior clear space on sagittal views (Fig 1). Collection was extending anterior to the sternum into parities and there was evidence of mediastinal and hilar lymphadenopathy along with areas of pleural reaction (thickening in the left hemithorax) along the costal pleura (Fig 2). There was enlargement of subcarinal group of lymph nodes. Fat planes anterior to mediastinum under collection were maintained. Aspirate from swelling over manubrium sterni revealed positive culture for Mycobacterium tuberculosis and positive Zeil Neelsen staining for acid fast bacilli.

The child was started on antitubercular treatment with four drugs viz isoniazid, rifampicin, ethambutol and pyrazinamide. The child showed dramatic improvement within a month of institution of treatment. The pain and swelling subsided. She was switched over to continuation phase with two drugs isoniazid and rifampicin. 


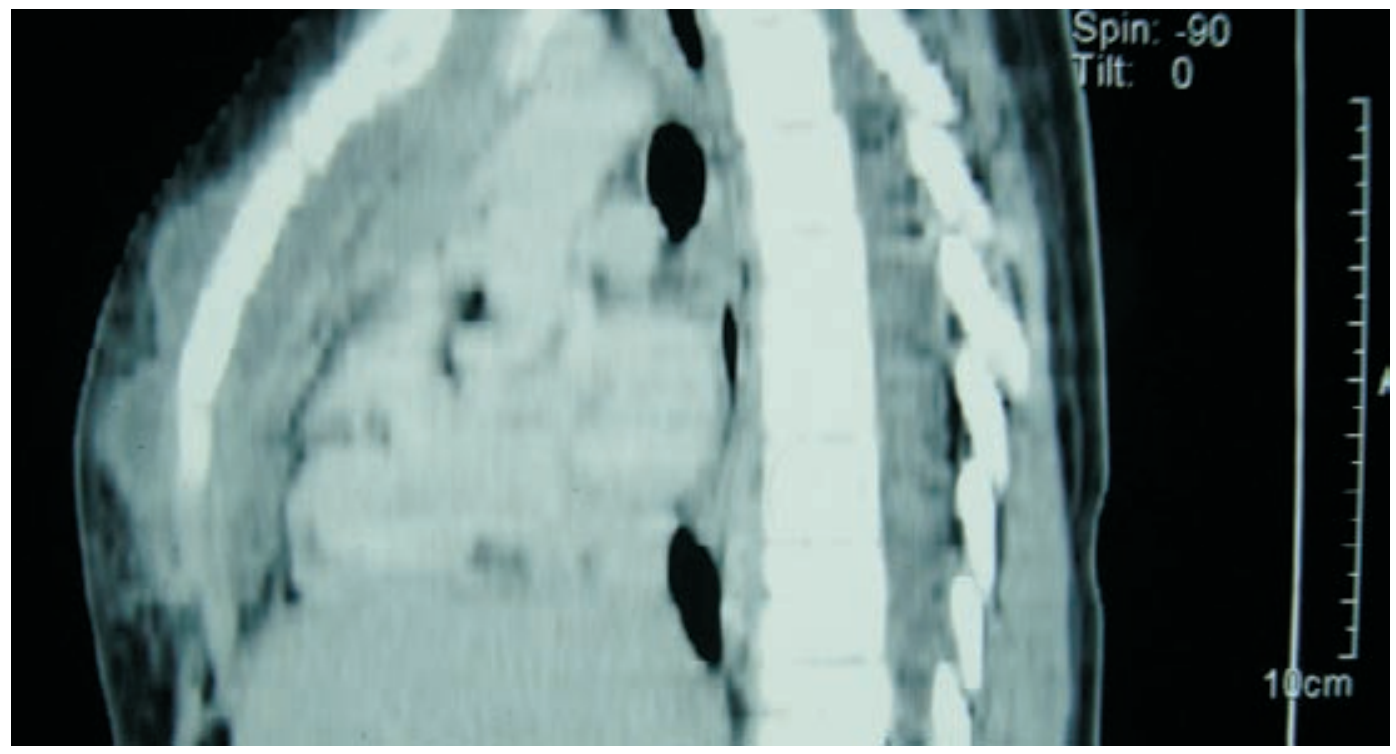

Figure 1: Sagittal MPR showing destruction of sternum with fluid collection obliterating the anterior clear space in chest and lying in contact with anterior mediastinal structure

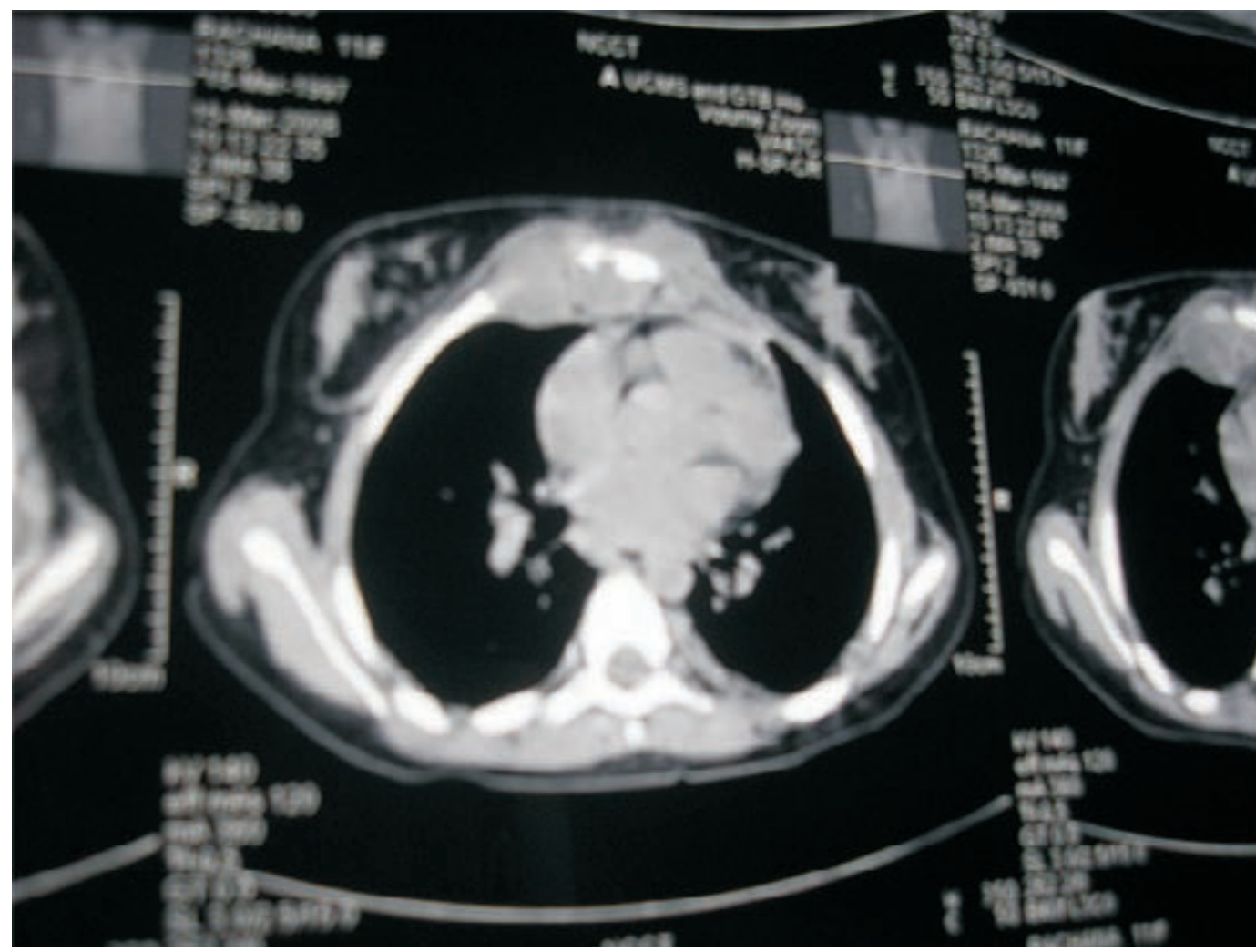

Figure 2: NCCT showing lytic destruction of sternum, costal end of ribs along with extrapleural thick collection within soft tissue.

\section{Discussion}

Tuberculous involvement of the sternum is rare presentation of extrapulmonary tuberculosis[3]. The diagnosis is often delayed because osseous tuberculosis is a paucibacillary lesion and smears are often negative. Localised pain, fever and weight loss are common modes of presentation. Our patient presented with a painful swelling over anterior chest wall as initial 
manifestation of sternal tuberculosis.

There are few reports of tuberculosis infection of sternum. Out of 20 cases of sternal osteomyelitis reviewed by McLellan et al, only 2 were children[4]. The earliest age at presentation has been 9 months, described in a Japanese infant[5]. Ray et al described sternal involvement in a case of disseminated tuberculosis in a child[6]. Tuberculosis of sternum may complicate thalassemia and HIV $[7,8]$. Spontaneous fracture of sternum with tuberculous infection has been reported [9].

Tubercular sternal osteomyelitis is usually caused by reactivation of latent loci formed during hematogenous or lymphatic dissemination of primary tuberculosis. In our case extrasternal involvement was suggested by presence of bilateral pleural effusion. Unlike pyogenic sternal infections characterized by more rapid and fulminant course, tubercular sternal osteomyelitis might present with sternal pain, painful swelling, cold abscess and parasternal sinus.

The gold standard for diagnosis of osseous tuberculosis is culture of Mycobacterium tuberculosis which was positive in our case. Our case also had positive Zeil Neelsen staining for acid fast bacilli but this does not differentiate between tuberculous and non tuberculous mycobacteria[10,1 1]. The advent of DNA detection via PCR has improved the diagnostic accuracy for exclusion of non tuberculous mycobacteria. The extent of bony lesion and degree of soft tissue involvement may be defined better by CT scan. MRI is helpful in differentiating between abscess and granulation tissue.

There is still no consensus on the optimal therapy of chest wall tuberculosis. Some experts favour a surgical treatment others argue that combination antitubercular chemotherapy should be the mainstay of treatment. Current recommendations for the treatment of sternal tuberculosis include a two month initial phase of isoniazid, rifampicin, ethambutol, pyrazinamide followed by a six to twelve months regimen of isoniazid and rifampicin. Our patient is responding well to medical treatment. However, close follow up is needed in these patients to determine whether surgical treatment will ultimately be needed.

This case illustrates the importance of high index of suspicion when evaluating a patient with swelling over chest wall. Early imaging and appropriate microbiological testing can avoid diagnostic delay.

\section{References}

1. Nicholson, R.A. Twenty years of bone and joint tuberculosis in Bradford. J Bone \& Jt. Surg Br. 1974; 56-B: 760-765.

2. Sharma S, Juneja M, Garg A. Primary tubercular osteomyelitis of the sternum. Indian J Pediatr 2005; 72:709-710.

3. Kelly CA, Chetty MN. Primary sternal osteomyelitis. Thorax 1985; 40: 872-873.

4. McLellan DGJ, Philips KB, Corbett CE, Bronze MS. Sternal osteomyelitis caused by Mycobacterium tuberculosis : case report and review of literature. Am J Med Sci 2000; 31 9: 250-254.

5. Kato Y, Horikawa Y, Nishimura Y, Shimoda H, Shigeto E, Ueda K. Sternal tuberculosis in a 9month-old infant after BCG vaccination. Acta Paediatr 2000; 89: 1495-1 497.

6. Ray $M$, Kataria $S$, Singhi P. Unusual presentation of disseminated tuberculosis. Indian Pediatr 2002; 39: 88-91.

7. Kataria S.P, Avasthi R. Sternal tuberculosis in combination with thalassemia. J Assoc Phys India. 1993; $41: 472$.

8. Martors JA, Olm M, Miro JM, Mallotas J, Letang E, Brancos MA, et al. Chondrocostal tuberculosis in 2 heroin addicts infected with human immunodeficiency virus. Med Clin [Barc] 1989; 93: 467-470.

9. Watts RA, Paice EW, White AG. Spontaneous fracture of the sternum and sternal tuberculosis. Thorax 1987; 42: 984-985.

10. Rooney JJ Jr, Crocco JA, Kramer S, Lyons HA. Further observations on tuberculin reactions in active tuberculosis. Am J Med 1976; 60:517-522.

11. Glassroth J. Diagnosis of tuberculosis. In: Tuberculosis: A Comprehensive International Approach. Reichman LB, Hershfield ES. Ed. New York, Marcel Dekker 1993 pp 149-165. 